\title{
5 Chancen und Grenzen in der Arbeit mit Inhaftierten des Jugendstrafvollzugs - Forensische Projektarbeit in der Jugendanstalt Schleswig/Teilanstalt Neumünster
}

Denis Köhler, Silvia Müller und Günter Hinrichs

5.1 Universitätsklinik für Kinder- und Jugendpsychiatrie und Psychotherapie des Zentrums für Integrative Psychiatrie Kiel (ZIP gGmbH)

Das Einzugsgebiet der Klinik für Kinder- und Jugendpsychiatrie (Direktorin: Prof. Dr. Dr. Lioba Baving) umfasst den Großraum Kiel mit ca. 300.0oo Einwohnern (Vollversorgung), bei bestimmten Fragestellungen gilt die Zuständigkeit für das gesamte Bundesland Schleswig-Holstein, welches im nördlichsten Teil Deutschlands liegt. Der stationäre Bereich besteht aus zwei Jugendlichen- und zwei Kinderstationen, wobei auch unter geschützten (geschlossenen) Bedingungen gearbeitet werden kann. Das Behandlungsangebot erstreckt sich auf alle kinder- und jugendpsychiatrischen Störungsbilder, auch akute psychotische (bei Selbst-und/oder Fremdgefährdung) Störungen können behandelt werden. Für geistig behinderte Patienten besteht allerdings kein spezifisches Behandlungsangebot, und bei Bedarf wird in diesen Fällen mit spezialisierten Einrichtungen kooperiert. In der Institutsambulanz werden 
5 Chancen und Grenzen in der Arbeit mit Inhaftierten des Jugendstrafvollzugs Forensische Projektarbeit in der Jugendanstalt Schleswig/Teilanstalt Neumünster

v.a. diagnostische Fragestellungen (z.B. ADHS) bearbeitet und ggf. psychotherapeutische Interventionen eingeleitet. Es besteht auch die Möglichkeit für Eltern von Kindern mit einer ADHS, an einem Trainingsprogramm teilzunehmen. Zusätzlich gibt es eine mit zwölf Behandlungsplätzen ausgestattete Tagesklinik für primär verhaltensgestörte Kinder im Altersbereich von sieben bis elf Jahren (ADHS und Störung des Sozialverhaltens). Neben dieser Versorgungsarbeit ist die Klinik durch die Zugehörigkeit zur Christian-AlbrechtsUniversität zu Kiel in der Forschung und Lehre tätig, vorwiegend im forensischen und neuropsychologisch-psychiatrischen Bereich.

\subsection{Die Jugendanstalt Schleswig/Teilanstalt Neumünster}

In der Jugendanstalt Schleswig sind Häftlinge mit eher kurzen Haftstrafen (mehrheitlich bis zu zwei Jahren) in einem Wohngruppenvollzug untergebracht (s. Box). Diese Anstalt verfügt über 73 Haftplätze im geschlossenen und über zehn im offenen Bereich. In der 8okm entfernten Teilanstalt Neumünster befindet sich die zentrale Aufnahme-Einrichtung für alle jugendlichen und heranwachsenden Straftäter des Landes Schleswig-Holstein. Dort sind ungefähr 105 Häftlinge im geschlossenen Vollzug untergebracht. Je nach Belegungsstand kann die Zahl von Inhaftierten jedoch unter oder über der offiziellen Anzahl von Haftplätzen liegen. Der Ausländeranteil liegt in beiden Anstalten zwischen $30-40 \%$. Das Durchschnittsalter der Inhaftierten beträgt ca. 19 Jahre bei einer Alterspanne von 14-25 Jahren. Die jugendlichen Straftäter verweilen durchschnittlich zwölf Monate im Jugendvollzug.

\section{Jugendanstalt Schleswig \\ - eher kurze Haftstrafen \\ - Wohngruppenvollzug}

\section{Teilanstalt Neumünster}

- Zentrale Aufnahme-Einrichtung für alle Jugendlichen in Schleswig-Holstein

- eher lange Haftstrafen

\section{Gesamtüberblick}

Haftplätze Schleswig: 73 Neumünster: 105

Offen: 10

Ausländeranteil: ca. $30-40 \%$

Alter: ca. 19

Verweildauer: ca. 12 Monate

Jugendliche: $14-18$ Jahre

Heranwachsende: 18-21 Jahre

Altersspanne: ab 14 Jahre bis ca. 25 Jahre 
Anzumerken ist in diesem Zusammenhang, dass in der Bundesrepublik Deutschland 14-18-Jährige als Jugendliche und 18-21-Jährige als Heranwachsende bezeichnet werden. Bei letzterer Gruppe, den Heranwachsenden, kann bei festgestellten Reifedefiziten eine Verurteilung nach dem Jugendgerichtsgesetz ( $\$ 105$ JGG) erfolgen.

\subsection{Entwicklung der forensischen Projektarbeit}

Seit 1989 besteht die bundesweit einmalige Zusammenarbeit zwischen der Universitätsklinik für Kinder- und Jugendpsychiatrie und -psychotherapie des Zentrums für Integrative Psychiatrie (ZIP gGmbH) Kiel und der Jugendanstalt Schleswig/Teilanstalt Neumünster (s. Box). Ausgangspunkt dafür waren einerseits die Erfahrungen der damaligen Universitätsklinik in der gutachterlichen Tätigkeit sowie vereinzelter Kontakte zu ehemals begutachteten Straftätern. Darüber hinaus fanden in Einzelfällen ambulante und stationäre Behandlungen statt (Schütze u. Hotamanidis 1989). Ein Anliegen seitens der Jugendanstalt war, eine angemessene Form der Betreuung für Inhaftierte mit aggressiven Straftaten und/oder Sexualdelikten zu finden, die der herkömmliche Jugendvollzug bisher nicht ermöglichte. Zielvorstellungen waren dabei eine intensive Auseinandersetzung mit der Tat und die Bearbeitung spezifischer Störungsanteile bei eventuell vorliegenden psychischen Störungen.

\section{Geschichte, Entwicklung der Zusammenarbeit und Einflüsse des Projektes}

- Exemplarische Behandlung nach Begutachtung

- intensivere Zusammenarbeit KiJu Kiel und JA Neumünster

- 1989 Beginn des Drittmittelprojekts, finanziert durch das Justizministerium des Landes Schleswig-Holstein

\section{Aus der Zusammenarbeit entstanden u.a.:}

- Sankelmarker Thesen zur Forensischen Psychotherapie (Beier u. Hinrichs 1995)

- Lehrbuch Forensische Psychiatrie und Psychologie des Kindes- und Jugendalters (Lempp et al. 1999)

- Täterverhalten und Persönlichkeit (Müller et al. 2005)

In den mittlerweile 18 Jahren der Kooperation zwischen den Institutionen hat sich eine sehr konstruktive Zusammenarbeit entwickelt. Über das rein psychotherapeutische Angebot hinaus entstanden im Verlauf zusätzliche Tätigkeitsfelder, die nachfolgend inhaltlich präzisiert werden (s. Box).

Die Klinik hat das psychotherapeutische Angebot auf die spezielle Klientel (tat- und störungsspezifisch) und die Bedürfnisse des Vollzugs abgestimmt (vgl. Hinrichs et al. 2004; Müller et al. 2007). Durch die wissenschaftliche Be- 
5 Chancen und Grenzen in der Arbeit mit Inhaftierten des Jugendstrafvollzugs Forensische Projektarbeit in der Jugendanstalt Schleswig/Teilanstalt Neumünster

gleitforschung wird eine Qualitätssicherung der therapeutischen Arbeit erreicht, die beiden Institutionen einen aktuellen Wissensstand verschafft (vgl. Köhler 2004; Köhler et al. 2007).

\section{Die therapeutischen Angebote}

- Einzelpsychotherapie

- Tataufarbeitung

- Vollzugsplankonferenzen (JA Schleswig)

- Erster Montag im Monat Konferenz (EMOMO; Teilanstalt Neumünster)

- Gespräche mit Bezugsbediensteten und Abteilungsleitern

- Krisenintervention (bei Selbst- oder Fremdgefährdung)

- Beratung des Anstaltsarztes/ärztin bei medikamentöser Behandlung von kinder- und jugendpsychiatrischen Störungsbildern (Konsiliartätigkeit)

\section{Schwerpunkt:}

Einzelpsychotherapie, ca. 30 Patienten/Klienten, einmal wöchentlich

\subsubsection{Psychotherapeutische Konzepte}

Die Mitarbeiter des Projektes sind keiner spezifischen psychotherapeutischen Schule verpflichtet und weisen dementsprechend unterschiedliche therapeutische Ausbildungen auf. Das Behandlungskonzept orientiert sich pragmatisch am größtmöglichen Nutzen im Einzelfall (multimodal und eklektisch; s. Box). Zum Einsatz kommen primär verhaltenstherapeutische, psychodynamische und systemische Interventionen, wobei die Basis des therapeutischen Vorgehens das große Spektrum spezialisierter deliktorientierter Arbeitsweisen und rückfallpräventiven Ansätze bildet, z.B. das Sex-Offender-Treatment-Programm (Berner u. Becker 20o1) oder Anti-Agressivitäts-Trainings (u.a. Weidner 1990). Insgesamt ist das psychotherapeutische Konzept am Grundsatz orientiert, dass Tätertherapie primär dem Opferschutz dient (vgl. auch Müller et al. 2007).

\section{Psychotherapeutisches Konzept}

- nach Probekontakten erfolgt eine Therapie

- Mitarbeiter des Projektes weisen unterschiedliche therapeutische Ausbildungen auf

- Behandlungskonzept: multimodal, eklektisch und am Einzelfall orientiert

- überwiegend kognitiv-verhaltenstherapeutische, psychodynamische und systemische Interventionen

- Basis bilden spezielle deliktorientierte Arbeitsweisen/Konzepte rückfallpräventiver Therapien (z.B. Sex Offender Treatment Programm, Anti-Aggressivitäts-Training) 


\section{Einzelpsychotherapie}

Von den Mitarbeitern und Mitarbeiterinnen in den beiden Anstalten werden ca. 30 Klienten/Patienten regelmäßig einzelpsychotherapeutisch versorgt. Vorwiegend handelt es sich hierbei um Sexual- und Gewaltstraftäter. Allerdings bestehen auch Therapieplätze für Insassen mit anderen Delikten.

Nachdem die Inhaftierten einen schriftlichen Antrag auf psychologische Gespräche gestellt haben, wird zunächst in einem orientierenden Vorgespräch die Indikation und Dringlichkeit einer therapeutischen Maßnahme festgestellt. Weiter erhalten die Antragsteller Informationen über die Rahmenbedingungen (z.B. Schweigepflicht, Ablauf und Inhalte der Gespräche). Anhand dieser ersten klinischen Einschätzung werden die Klienten/Patienten entweder auf eine Warteliste gesetzt oder sofort behandelt. Anschließend erfolgt die sogenannte „probatorische Phase“ oder „Kennenlernphase“ genannt, welche meist fünf Sitzungen umfasst. In dieser Zeit werden die Anliegen, Beschwerden, Probleme, das Delikt und die Therapieziele des Häftlings systematisch erfasst. Zusätzlich findet neben einer standardisierten psychodiagnostischen Untersuchung (Intelligenz, Persönlichkeitseigenschaften, psychische Störungen) auch ein Screening der Therapiemotivation statt. Dafür werden sowohl semi-strukturierte klinische Interviews (Strukturiertes Klinisches Interview für DSM-IV; Wittchen et al. 1997; Psychopathy Checklist, Hare 2003) als auch Selbstbeurteilungsverfahren verwendet (z.B. Inventar Klinischer Persönlichkeitsakzentuierungen, NEO-Fünf-Faktoren Inventar). In Abbildung 3 ist dieser diagnostische Ablauf dargestellt. Sollte nach dieser Phase eine anschließende psychotherapeutische Behandlung indiziert sein, wird diese ggf. einzelfallorientiert evaluiert (Hinrichs et al. 2004).

Die inhaltlichen Schwerpunkte der Behandlung sind in der Box unten kurz dargestellt. Neben einer Auseinandersetzung mit der Tat werden v.a. Aspekte des Empathietrainings und der Ärger/Aggressionskontrolle bearbeitet sowie die sozialen Kompetenzen gestärkt. Des Weiteren werden auch störungsspezifische Interventionen zur Linderung psychiatrischer Symptome durchgeführt. Eine ausführliche Darstellung des Therapiekonzeptes der Arbeitsgruppe haben Müller et al. (2007) im Forum Strafvollzug veröffentlicht.

\section{Schwerpunkte der forensischen Psychotherapie:}

- Tatverarbeitung

- soziale Kompetenztrainings/soziale Bewältigungstechniken

- Entspannungsverfahren (z.B. Autogenes Training)

- Empathietraining, Ärgerkontrolle

- Störungsspezifische Therapie (Linderung psychiatrischer Symptome)

n kognitive Umstrukturierung (Persönlichkeitsakzentuierungen/Störungen) 
5 Chancen und Grenzen in der Arbeit mit Inhaftierten des Jugendstrafvollzugs Forensische Projektarbeit in der Jugendanstalt Schleswig/Teilanstalt Neumünster

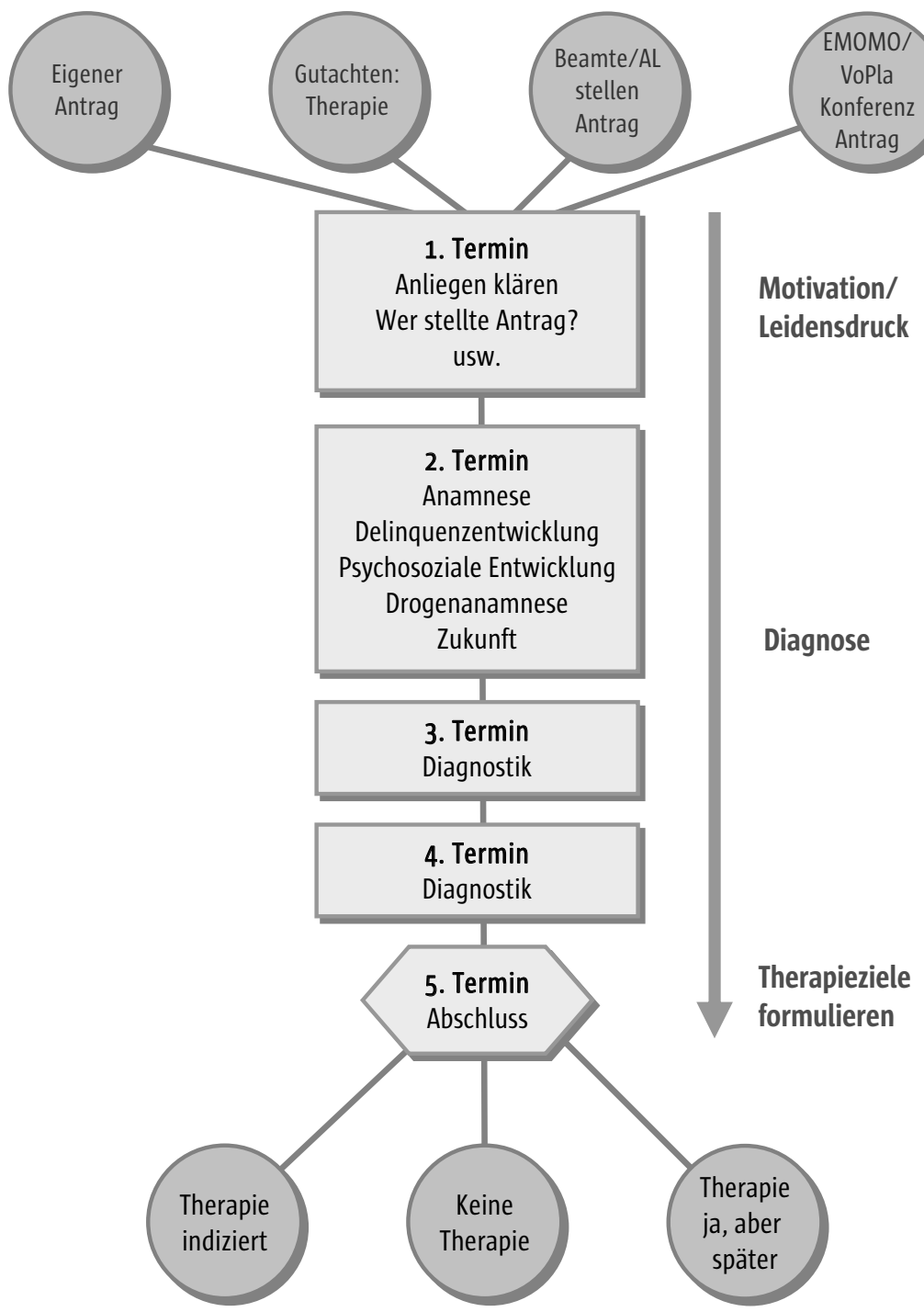

Abb. 3 Intramurale Psychotherapie mit jugendlichen Inhaftierten in der JA Münster

\section{Gruppenpsychotherapie}

Zum gegenwärtigen Zeitpunkt wird kein psychotherapeutisches Cruppenprogramm angeboten. Es besteht für die jugendlichen und heranwachsenden Gefangenen aber die Möglichkeit, an einem Anti-Aggressionstraining (AAT) teilzunehmen. Dieses wird jedoch von einer anderen sozialen Einrichtung durchgeführt. Zwischen den Behandelnden der verschiedenen Institutionen 
besteht allerdings ein regelmäßiger fachlicher Austausch, so dass die einzelnen Therapieangebote sinnvoll auf einander abgestimmt werden.

\subsubsection{Krisenintervention}

Durch Haft, existentielle Notlagen oder deliktspezifische Erschütterungen verursachte suizidale Krisen werden nach Bedarf mit den in Frage kommenden Inhaftierten bearbeitet. Dies geschieht in Absprache mit der Abteilungsleitung, den Vollzugsbediensteten und dem Anstaltsarzt/der Anstaltsärztin.

\subsubsection{Aspekte der Schweigepflicht}

Der Informationsaustausch zwischen den Mitarbeitern des Projektes und den im Vollzug Beschäftigten erfolgt lediglich unter ausdrücklicher Entbindung von der Schweigepflicht. Bei jeder Kontaktaufnahme wird dieses Problemfeld mit den Gefangenen bzw. Klienten ausführlich erörtert. Die Sensibilität gestaltet sich recht unterschiedlich, unter anderem in Abhängigkeit von Alter und Persönlichkeit. Stellungnahmen zu anstehenden Vollzugslockerungen oder der vorzeitigen Entlassung werden in der Regel nicht abgegeben, sondern einem forensischen Gutachter überlassen. Dem Vollzug werden lediglich das therapeutische Vorgehen, Status der Behandlung (Erstkontakt, Probephase oder Behandlung) und die Anzahl der stattgefundenen Kontakte mitgeteilt.

\subsubsection{Medikamentöse Behandlung}

Die ärztlichen Mitarbeiter der Klinik für Kinder- und Jugendpsychiatrie und -psychotherapie Kiel stehen der Anstaltsärztin bzw. dem Anstaltsarzt bei Bedarf konsiliarisch und beratend zur Seite, wenn die Behandlung von kinderund jugendpsychiatrischen Störungen erforderlich ist.

\subsubsection{Gesprächskontakte mit Angehörigen der Inhaftierten}

Um eine stabile und langfristige Änderung über die Haftentlassung hinaus zu ermöglichen, sind Gespräche mit Familien und anderen wichtigen Bezugspersonen oft unverzichtbar. Diese werden in Absprache und teilweise auch in Anwesenheit der Jugendlichen oder Heranwachsenden geführt. Allerdings gestaltet sich die Einbeziehung von Bezugspersonen durch die intramuralen Rahmenbedingungen (z.B. Sicherheitsaspekte) und die oft sehr problematischen Familienverhältnisse der Inhaftierten als organisatorisch sehr aufwendig und meist wenig tragfähig. 
5 Chancen und Grenzen in der Arbeit mit Inhaftierten des Jugendstrafvollzugs -

Forensische Projektarbeit in der Jugendanstalt Schleswig/Teilanstalt Neumünster

\subsubsection{Ambulante psychotherapeutische Weiterbehandlung}

In Einzelfällen (durchschnittlich zweimal pro Jahr) werden Gefangene nach ihrer Entlassung in der Klinik für Kinder- und Jugendpsychiatrie Kiel ambulant psychotherapeutisch weiter behandelt. Die Grundlage für ein derartiges Angebot stellt zumeist eine Bewährungsauflage bei einer vorzeitigen Entlassung dar und beinhaltet daher eine enge Kooperation mit der Bewährungshilfe und dem zuständigen Gericht.

\subsubsection{Schwierigkeiten und Grenzen intramuraler forensischer Psychotherapie}

Jugendliche und heranwachsende Straftäter sind deutlich psychisch belastet und weisen eine hohe Prävalenz von psychischen Störungen auf. Zusätzlich liegen bei ihnen häufig Substanzmissbrauch bzw. -abhängigkeit vor (Köhler 2004; Köhler et al. 2007). Deshalb erscheinen therapeutische Interventionen bei dieser Klientel nicht nur aus legalprognostischen Gründen, sondern auch aus psychologisch-psychiatrischer Sicht dringend notwendig. Jedoch weist der Kontext Strafvollzug einige Schwierigkeiten und manchmal sogar Grenzen für die intramurale Psychotherapie auf, die im Folgenden exemplarisch diskutiert werden (vgl. auch Box unten).

\section{Schwierigkeiten und Grenzen}

- institutionelle Rahmenbedingungen (justizieller Kontext)

n teilweise defizitäre pädagogische Weiterbildung der Vollzugsbediensteten

- subkulturelle Einflüsse (Gewaltanwendung als adäquates Mittel)

- delinquente Peergroup/fehlende prosoziale Modelle

- Zuordnung der Klienten nach Delikt, weniger nach psychischer Störung

- Therapiemotivation (extrinsisch vs. intrinsisch)

- Zielsetzungen der Therapie (Legalverhalten als einziges Kriterium?)

- Modalitäten der Schweige/Offenbarungsverpflichtung

- Spannungsfeld Öffentlichkeit, therapeutische Dyade und Vollzug

Psychotherapie bleibt letztendlich von konsiliarischem Charakter (einmal wöchentlich).

Eine Jugendanstalt gilt als totalitäre Institution mit wenigen Freiheitsgraden für die Inhaftierten sowie einer negativ geprägten Subkultur und beschränkt dadurch die Möglichkeiten einer prosozialen Verhaltensmodifikation. Die Zusammenarbeit mit dem Allgemeinen Vollzugsdienst, der den intensivsten sozialen Kontakt mit den Insassen pflegt, ist unverzichtbarer Bestandteil der Projektarbeit, aber in Einzelfällen durchaus problematisch. Häufig mangelt es an pädagogischen Fort-/Weiterbildungen für die Vollzugsbediensteten, die einerseits Sicherheitsaufgaben zu bewältigen haben, an- 
dererseits jedoch auch den pädagogischen Anforderungen eines Behandlungsvollzuges gerecht werden sollen. Darüber hinaus bildet sich bei den Inhaftierten eine Subkultur heraus, die Gewalt als adäquates Mittel legitimiert. Delinquente Peergroups und das damit einhergehende Fehlen prosozialer Modelle führen insgesamt zu einem eher geringen psychotherapeutischen Klima.

Aus dem psychotherapeutischen Blickwinkel erscheint die Einhaltung der Schweigepflicht als schwierig, v.a. falls sich während der Gespräche deliktoder störungsspezifische Sachverhalte ergeben, die eventuell auf ein erhöhtes Rückfallrisiko oder eine akute Selbst- oder Fremdgefährdung hinweisen. Im Einzelfall werden solche Vorkommnisse im Rahmen der regelmäßigen Projektbesprechungen diskutiert und hinsichtlich des weiteren Vorgehens koordiniert.

Die Klienten werden vom Vollzug - meist im Rahmen der Aufnahmeuntersuchung - primär nach Art des Deliktes (z.B. Gewaltstraftat, Sexualdelikt, Eigentumsdelikt) und nicht entsprechend ihrer psychischen Problematik für therapeutische Maßnahmen vorgeschlagen. Dieses Vorgehen widerspricht dem traditionellen psychotherapeutischen Verfahren, welches sich bei einer Therapieindikation eher an den zugrunde liegende psychischen Störungen orientiert. Nicht selten erscheinen die Insassen auch wenig intrinsisch motiviert und erhoffen sich aus der Teilnahme an einer Psychotherapie Vorteile im Vollzug. Eine Fehlzuordnung von Patienten/Klienten ist daher in Einzelfällen nicht auszuschließen.

Ein weiterer schwieriger Aspekt ist die Festlegung des Kriteriums für eine erfolgreiche therapeutische Intervention. Die Linderung psychiatrischer Symptome und des Leidensdrucks der Klienten scheint für die forensische Psychotherapie alleine nicht zu gelten. Vielmehr orientiert man sich hauptsächlich an der Rückfallrate als Validitätskriterium. Dies wird jedoch offensichtlich durch viele Einflussfaktoren moderiert (z.B. psychosoziale Lebensbedingungen bei einer Haftentlassung) und liegt damit oftmals außerhalb des Einflusses intramuraler psychotherapeutischer Möglichkeiten.

Bei all diesen aufgezeigten Schwierigkeiten und Grenzen muss jedoch abschließend die gute und konstruktive Zusammenarbeit zwischen der Klinik für Kinder- und Jugendpsychiatrie und Psychotherapie Kiel und der Jugendanstalt Schleswig/Teilanstalt Neumünster betont werden. Neben den genannten Problemen überwiegen die positiven Aspekte des Projekts deutlich. In den letzten Jahren hat die Zusammenarbeit zwischen der Klinik und der Jugendanstalt den Charakter eines Projekts nahezu verloren und sich quasi zu einer „Dauereinrichtung“ entwickelt. Als zusätzliche Erschwernis fehlt allerdings bislang eine längerfristige Finanzierung der Projektstellen, so dass jedes Jahr die Projektgelder erneut bewilligt werden müssen. 
5 Chancen und Grenzen in der Arbeit mit Inhaftierten des Jugendstrafvollzugs -

Forensische Projektarbeit in der Jugendanstalt Schleswig/Teilanstalt Neumünster

\subsubsection{Weitere Kooperationen zwischen den beiden Institutionen}

\section{Gesprächskontakte mit Vollzugsmitarbeitern/innen}

Zur Kontrolle und Validierung der therapeutischen Arbeit sowie der Begleitung des Vollzugsverlaufs führen die Projektmitarbeiter Gespräche mit den Vollzugsbediensteten, insbesondere mit der Abteilungsleitung. Dies findet in Absprache mit dem Klienten/Patienten unter Wahrung der Schweigepflicht statt.

\section{Erste Montag im Monat-Konferenz (EMOMO)}

Jeden ersten Montag im Monat (EMOMO) findet in der Teilanstalt Neumünster eine Konferenz statt, an der die Anstaltsleitung, die Abteilungsleiterinnen, die Bezugsbeamten und die Mitarbeiter der Klinik für Kinder- und Jugendpsychiatrie teilnehmen. In diesem Rahmen werden „problematische“ Häftlinge besprochen und deren Entwicklung(smöglichkeiten) bzw. Vollzugsplanung diskutiert. Das Anliegen der im Vollzug Tätigen war es, konkrete Hinweise für den Umgang mit diesen Häftlingen aus (psycho)therapeutischer Sicht zu bekommen, um so psychologische Aspekte bei der weiteren Vollzugsplanung berücksichtigen zu können. Für die Forensische Psychotherapie hat sich diese Konferenz ebenfalls als sehr hilfreich erwiesen, da den Projektmitarbeitern die alltäglichen Verhaltensweisen der Klienten/Patienten rückgemeldet werden, die außerhalb des psychotherapeutischen Settings liegen. Es werden nur Häftlinge besprochen, die sich vorher damit einverstanden erklären und die Projektmitarbeiter begrenzt von der Schweigepflicht entbinden. Konkrete Inhalte der psychotherapeutischen Gespräche aus dem explizit persönlichen Bereich werden allerdings nicht offenbart.

\section{Vollzugsplanungs-Konferenz (VoPla)}

Ähnlich der zuvor beschriebenen EMOMO findet auch in der Jugendanstalt Schleswig in einem etwa dreiwöchigen Rhythmus eine Besprechung über einzelne Inhaftierte statt. Gemeinsam reflektieren Anstaltsleitung, Abteilungsleitung und Mitarbeitern der hier vorgestellten forensischen Projektarbeit die Entwicklung der Inhaftierten im Vollzug und entwerfen pädagogische Ziele. Die relevanten Inhalte aus der Einzeltherapie werden dabei zuvor mit den Klienten/Patienten besprochen.

\section{Supervision der eigenen Projektmitarbeiter}

Die Mitarbeiter und Mitarbeiterinnen des Projektes haben zwischenzeitlich an einer forensischen Fallsupervision teil genommen. Einmal wöchentlich findet für alle Mitarbeiter der Klinik eine Therapiebesprechung (Balint-Gruppe) statt. Diese Supervisionsangebote dienen einerseits der psychotherapeutischen Qualitätssicherung und andererseits der Weiterbildung. 


\section{Fortbildungsveranstaltungen und Vorträge für die interne Weiterbildung des Vollzuges und der Vollzugsbediensteten}

In regelmäßigen Abständen bieten die Mitarbeiter des Projekts „Forensische Psychotherapie“ in Zusammenarbeit mit den beiden Anstalten zu den verschiedensten Themen Workshops, Seminare und Vorträge an. Es werden u.a. folgende Themen behandelt:

- Förderung im Vollzug von minderbegabten Gefangenen im Jugendstrafvollzug

- Umgang mit Sexualstraftätern im Jugendvollzug

- Einführung in den Beobachtungsbogen für Häftlinge

- Psychotherapie im Strafvollzug: zur Schnittstelle von Pädagogik und Psychotherapie

- Forschungsergebnisse zur psychischen Befindlichkeit und der Prävalenz psychischer Störungen bei jugendlichen und heranwachsenden Häftlingen

- Umgang mit „auffälligen“ Inhaftierten

\subsection{Wissenschaftliche Begleitforschung}

Von den Projektmitarbeitern werden in Kooperation u.a. mit der Universität Hamburg und Kiel verschiedene Forschungsprojekte durchgeführt (z.B. Tatverarbeitung, standardisierte psychologische Eingangsdiagnostik). In den letzten Jahren wurden zahlreiche wissenschaftliche Beträge zu unterschiedlichen Themen veröffentlicht: Psychotherapie im Strafvollzug, die Prävalenz psychischer Störungen bei Inhaftierten des Jugendvollzuges, die psychische Befindlichkeit und die Behandlungsvoraussetzungen im Sinne der Therapiemotivation, Täterverhalten und Persönlichkeit, Risiko- und Schutzfaktoren (Beier u. Hinichs 1995; Köhler 2004; Müller et al. 2005; Köhler et al. 2007; Köhler et al. in Druck). Die Ergebnisse der wissenschaftlichen Begleitforschung werden regelmäßig auf Kongressen, Kolloquien etc. präsentiert und veröffentlicht. Bei Interesse können diese beim Verfasser angefordert werden.

\subsection{Schlussfolgerungen}

Forensische Psychotherapie bewegt sich inhaltlich im Spannungsfeld zwischen der Bearbeitung von störungsspezifischen, tatbezogenen und legalprognostischen Aspekten. Der beschriebene therapeutische Ansatz soll den folgenden drei Bereichen bzw. Aspekten gerecht werden:

- Erstens besteht eine Indikation für eine forensisch-psychotherapeutische Tätigkeit aufgrund der hohen Prävalenz psychischer Auffälligkeiten (störungsspezifische Aspekte). Entsprechend gängiger multikausaler (bio-psycho-sozialer) Kriminalitätsmodelle sind aber zudem andere 
Variablen, wie z.B. die spezielle Lebenssituation oder situative Einflüsse, zu berücksichtigen (vgl. Köhler 2004), so dass ein entwicklungsbezogener und ressourcenorientierter Ansatz unabdingbar ist.

- Zum zweiten sind spezifisch forensische Therapieinhalte (u.a. Auseinandersetzung mit der Straftat, Verbesserung der Legalprognose) unter der Zielsetzung einer Tatverarbeitung im Verlauf der intramuralen Behandlung maßgeblich. Das Besondere der dargestellten Projektarbeit liegt sicherlich darin, die Behandlung in Abstimmung mit dem Vollzug weitestgehend adaptiv zu gestalten,

- so dass drittens dem kommunikativen Austausch mit der Anstalts/Abteilungsleitung ein hoher Stellenwert beizumessen ist.

\section{Literatur}

Beier KM, Hinrichs G (1995) Psychotherapie mit Straffälligen. Standorte und Thesen zum Verhältnis PatientTherapeut-Justiz. Stuttgart: Gustav Fischer.

Berner W, Becker KH (2001) "Sex Offender Treatment Programme“ (SOTP) in der Sozialtherapeutischen Abteilung Hamburg-Nesselstraße. In: Rehn G, Wischka B, Lösel F, Walter M (Hrsg.) Behandlung „gefährlicher Straftäter". Herbolzheim: Centaurus Verlag.

Hare RD (2003) Manual for the Revised Psychopathy Checklist ( $2^{\text {nd }}$ ed.). Toronto, ON, Canada: Multi-Health Systems.

Hinrichs G, Behnisch A, Krull K, Reimers S (2000) Therapiemotivation junger Strafgefangener. Zeitschrift für Kinder- und Jugendpsychiatrie und Psychotherapie 28: 255-262.

Hinrichs G, Köhler D, Repp N (2004) Psychotherapie mit jugendlichen Tötungsdelinquenten. Psychotherapie im Dialog 2: 144-149.

Köhler D (2004) Psychische Störungen bei jungen Straftätern. Eine Untersuchung zur Prävalenz und Struktur psychischer Störungen bei neu inhaftierten Jugendlichen und Heranwachsenden in der Jugendanstalt Schleswig. Hamburg: Verlag Dr. Kovac.

Köhler D, Hinrichs G, Baving L (in Druck) Therapiemotivation, Psychische Belastung und Persönlichkeit bei Inhaftierten des Jugendvollzuges. Zeitschrift für Klinische Psychologie und Psychotherapie, Heft 4.

Köhler D, Hinrichs G, Huchzermeier C (2004) Zur psychischen Belastung von jugendlichen und heranwachsenden Häftlingen (gemessen mit der SCL-90-R). Recht \& Psychiatrie 3: 138-142.

Köhler D, Müller S, Hinrichs G (2007) Psychische Störungen bei Inhaftierten des Jugendstrafvollzuges. Zeitschrift für Jugendkriminalrecht und Jugendhilfe 3: 253-259.

Lempp R, Schütze G, Köhnken G (1999) Forensische Psychiatrie und Psychologie des Kindes- und Jugendalters. Darmstadt: Steinkopf.

Müller S, Köhler D, Hinrichs G (2005) Täterverhalten und Persönlichkeit. Eine empirische Studie zur Anwendbarkeit der Tathergangsanalyse in der Forensischen Psychologie und Psychiatrie. Frankfurt: Verlag für Polizeiwissenschaft.

Müller S, Köhler D, Hinrichs G (2007) Psychotherapeutische Behandlung und Betreuung inhaftierter Gewaltund Sexualstraftäter in der Jugendanstalt Schleswig/Teilanstalt Neumünster Forum Strafvollzug. Zeitschrift für Strafvollzug und Straffälligenhilfe 4: 156-162.

Schütze G, Hotamanidis S (1989) Psychotherapie mit jugendlichen Straftätern - eine Fallstudie. In: Du Bois R (Hrsg.) Praxis und Umfeld der Kinder- und Jugendpsychiatrie. Bern: Huber.

Weidner I (1990) Das Anti-Aggressivitäts-Training für Gewalttäter. Ein deliktspezifisches Behandlungsangebot im Jugendvollzug. Bonn: Forum.

Wittchen H-U, Wunderlich U, Gruschwitz S, Zaudig M (1997) SKID-I. Strukturiertes Klinisches Interview für DSMIV. Achse I: Psychische Störungen. Interviewheft. Göttingen: Hogrefe.

Wittchen H-U, Zaudig M, Fydrich T (1997) SKID. Strukturiertes Klinisches Interview für DSM-IV. Achse I und II. Handanweisung. Göttingen: Hogrefe. 